BLS 33, No 1 2007. DOI: http://dx.doi.org/10.3765/bls.v33i1.3531

(published by the Berkeley Linguistics Society and the Linguistic Society of America)

\title{
Variation in Voice Onset Time of Stops: The Case of Chinese Korean*
}

WENHUA JIN

University of Texas at Arlington

\section{Introduction}

Despite the abundant research on the Korean language spoken in the Republic of Korea, other Korean varieties spoken in the rest part of the world, especially those spoken in China (hence Chinese Korean), have not received much attention in the linguistic literature.

Considering the relative geographic isolation of the Korean communities in China from Korea and the overall differences in the linguistic, political, and socio-cultural atmosphere between China and Korea, studies on Chinese Korean may reveal important patterns about Korean language variation and change, and also about language variation and change in general under eastern context. As in the case of Brazilian Portuguese where Guy (1981) found that the language has acquired many unique characteristics so that today it is quite different from European Portuguese in many aspects, Chinese Korean, after more than a century's acculturation with the Chinese mainstream Han culture, may manifest independent patterns of variation and change. Alternatively, it is also possible that Chinese Korean undergoes similar patterns of variation and change as those of Korea due to various internal and external factors.

Indeed, as revealed in this study, Chinese Korean manifests similar diachronic shift in voice onset time (VOT) as Seoul Korean reported in Silva $(2004,2006)$. More specifically, this study on Chinese Korean stops indicates that while older people maintain clearer distinction in VOT to mark the three different types of stops, younger speakers born in 70s and 80s no longer mark the lax and aspirated stops with the difference in VOT. Instead, Fundamental Frequency (F0) and intensity build-up are consistently employed to implement the phonological distinction.

\footnotetext{
* This study is made possible with the support of 2006-2007 International Fellowship granted to the author by American Association of University Women.
} 
Wenhua Jin

\section{Background \\ 1.1. The Korean Chinese}

With about 2 million people, the Korean people living in China today form the thirteenth largest population among the 55 minorities in China. The majority live in the three northeastern provinces in China: Hēilóngjiāng, Jílín, and Liáoníng. They are immigrants or descendents of immigrants from the Korean Peninsula, who formed different Chun (village) according to their different hometown Gwun (county) on the Korean Peninsula. Thus, the original features of their dialects were often maintained in the relatively homogenous villages in China (Zhao and Xuan 1986).

However, the current situation of Chinese Korean is more complicated. Due to the political relationship with North Korea, which uses the variety spoken in Phyengyang as the standard, language policies by the Chinese government often followed those of the North Korea especially in the 1960s (Cui and Quan 1993). Also, with the development of modern transportation and economy, the former relatively stable population became more mobile. On one hand, there is language contact between Chinese and Korean, and on the other hand, there is dialect contact between different varieties of Korean, especially since China's reform and opening-up. Thus, today's Chinese Korean preserves some original Korean forms (Zhao and Xuan 1986), manifesting meanwhile some innovations that may be explainable in terms of internal variation or various levels of contact (Silva and Jin 2006).

\subsection{Related Studies on Korean VOT}

Korean has been considered as typologically unique for its three-way-contrastive stop consonants: tense, lax, and aspirated. Studies on VOT of these stops of standard (Seoul) Korean suggest a diachronic shift in VOT values and a change in the phonological role played by VOT (Silva 2004, 2006). The landmark work of Lisker and Abramson (1964) is the first to reveal the existence of an overlap in VOT between tense and lax Korean stops, though they both can be distinguished from that of aspirated stops. This finding is confirmed in further studies by $\mathrm{C}-\mathrm{W}$ Kim (1965), Han and Weitzman (1965), Han and Weitzman (1970), Abramson and Lisker (1971). Follow-up studies in the 90s by Silva (1992), Cho (1996), and Han (1996), however, show that the observed overlap has been replaced with a clear distinction in VOT with the lax stops being more aspirated, thus confirming the major role of VOT in marking the phonological distinction of the three types of stops. The most recent studies by Silva $(2004,2006)$ reveal that many Seoul Korean speakers born after 1965 neutralize the VOT differences between lax and aspirated stops.

Similar study on Chinese Korean, extremely scant as it is, reveals a different pattern of variation. Zheng and Li's (2005) investigation on six young Korean Chinese in Yánbiān University indicates a tendency of merging in VOT between lax and tense stops, though that of aspirated stops remains distinct from both lax and tense stops. If this is true, then the three-way distinction in Korean VOT is 
shifting to a two-way distinction, albeit by two different paths: in Seoul Korean the merger is VOT between lax and aspirated stops while in Chinese Korean it is between the tense and lax stops.

Whether this VOT shift also occurs in other varieties of Chinese Korean among different generations remains a topic meriting further systematic study. Thus, the purpose of this study is to investigate the VOT in stops of Chinese Korean to capture and better understand the patterns of Korean language variation and change.

\section{Methodology}

\subsection{Subjects}

Subjects for this study are 22 native Chinese Korean speakers from the Korean community of Shěnyáng, China, ranging in age from 20 to 72, including 10 males and 12 females, with four people in each decade (two people for 60s). To avoid any potential variation due to dialect difference, only Phyengan dialect speakers were recruited through social networking approach. In demographic questionnaires, all the subjects self-claimed as speakers of Phyengan dialect with both parents being speakers of Phyengan dialect, and growing up in a community where Phyengan dialect dominated. They are all bilingual, speaking both Chinese and Korean. No subjects reported any difficulty in speech or hearing.

The subjects were asked to participate in an interview with the author for about one hour, which was recorded with a Dell laptop using Cool Edit 2000. An ATR20 Unidirectional Dynamic Microphone attached to the laptop was held by an assistant of the researcher at about $3 \mathrm{~cm}$ under the chin of the subject. The recordings were saved as wave files for further acoustic analysis with PRAAT.

\subsection{Materials}

The materials used here are a subset of the materials designed for a more comprehensive project. For this study, nine isolated three-syllable Korean words (Table (1)) were used with the nine target stops embedded in word initial position. Each of the target stop is immediately followed by the same vowel /a/. These three-syllable words were designed in the original project to be consistent with those three-syllable words where the behavior of the stops in real word-internal position is examined.

These words were printed in Korean script on a piece of paper mixed with other items for the larger project. Subjects were asked to read these words three times each at a normal, comfortable speed. For this study, the second readings were analyzed, yielding a total of 198 tokens.

\subsection{Data Measurement}

Acoustic measurements in this study include four categories: VOT of the target stop, F0 onset and offset, intensity build-up and vowel length of the immediately following vowel after the target stop. 
Wenhua Jin

(1)

Target Words Employed in This Study ${ }^{1}$

\begin{tabular}{|c|c|c|c|}
\hline \multirow{2}{*}{$\begin{array}{l}\text { Phonation } \\
\text { Type }\end{array}$} & \multicolumn{3}{|c|}{ Place of Articulation } \\
\hline & Labial & Alveolar & Velar \\
\hline Lax & $\begin{array}{l}\text { 바가지 } \\
\text { pa.ha.ci } \\
\text { "gourd" }\end{array}$ & $\begin{array}{l}\text { 다람줘 } \\
\text { ta.lam.cwi } \\
\text { "squirrel" }\end{array}$ & $\begin{array}{l}\text { 가죽신 } \\
\text { ka.cwuk.sin } \\
\text { "leather shoes" }\end{array}$ \\
\hline Tense & $\begin{array}{l}\text { 빠르다 } \\
\text { ppa.lu.ta } \\
\text { "fast" }\end{array}$ & $\begin{array}{l}\text { 따진다 } \\
\text { tta.cin.ta } \\
\text { 'come } \\
\text { unsewn' }\end{array}$ & $\begin{array}{l}\text { 까마귀 } \\
\text { kla.ma.kwi } \\
\text { "crow' }\end{array}$ \\
\hline Aspirated & $\begin{array}{l}\text { 파뿌리 } \\
\text { pha.ppu.li } \\
\text { 'shallot root' }\end{array}$ & $\begin{array}{l}\text { 타자기 } \\
\text { tha.ca.ki } \\
\text { "typewriter" }\end{array}$ & $\begin{array}{l}\text { 카나다 } \\
\text { kha.na.ta } \\
\text { 'Canada" }\end{array}$ \\
\hline
\end{tabular}

VOT was measured from the release point of stop closure to the point where the periodic striation and the first formant (F1) of the immediately following vowel start in the spectrogram. F0 onset was measured at the ending point of VOT, and F0 offset, at the point where the second formant (F2) sets off. Intensity build-up, defined here, is the measurement from the onset of voicing to the point of fullest development of amplitude in the wave form. In cases of uncertainty, intensity contour in the spectrogram was employed for reference. Vowel length was measured as the distance between onset of the voicing to the offset of F1 and F2 formants. All the measurements were subjected to statistical analysis with SPSS 13.0.

\section{Results}

\subsection{Patterns of VOT}

The examination on VOT values was conducted along two dimensions: place of articulation and phonation type. In terms of place of articulation, the mean VOT range has narrowed down over time.

In Figure (2), VOT values show a general tendency to decrease over time in all the three places of articulation categories, as captured by the trend lines. However, the three parallel lines suggest that the relative distinction between the three categories is maintained, confirming previous understanding that VOT tends to increase as the point of articulation moves toward the posterior region of the oral cavity (Ladefoged 2003, Silva 2006).

However, statistical analysis reveals that although the mean VOT values for labial and alveolar stops are not significant at $95 \%$ confidence interval (Mean $n_{\text {labial }}$ VOT $50.0 \mathrm{~ms}, M_{\text {alveolar }}$ VOT $\left.56.8 \mathrm{~ms}\right)$, Mean $_{\text {velar }}(68.9 \mathrm{~ms})$ is significantly different from both of the former $(p<0.05)$. This general pattern is maintained even when phonation types are taken into consideration. For example, mean VOT of $/ \mathrm{pp} /(14.1 \mathrm{~ms})$ is not significantly different from mean VOT of $/ \mathrm{tt} /(14.7 \mathrm{~ms})$, but

\footnotetext{
${ }^{1}$ Under Korean script are forms in Yale Romanization. The syllable boundaries are indicated by a period.
} 
that of $/ \mathrm{kk} /(25.3 \mathrm{~ms})$ is significantly different from those of the former two. One minor exception, though, is mean VOT of $/ \mathrm{t}^{\mathrm{h}} /$ is not significantly different form that of $/ \mathrm{k}^{\mathrm{h}}$.

(2)

Mean VOT Values for Different Places of Articulation (ms)

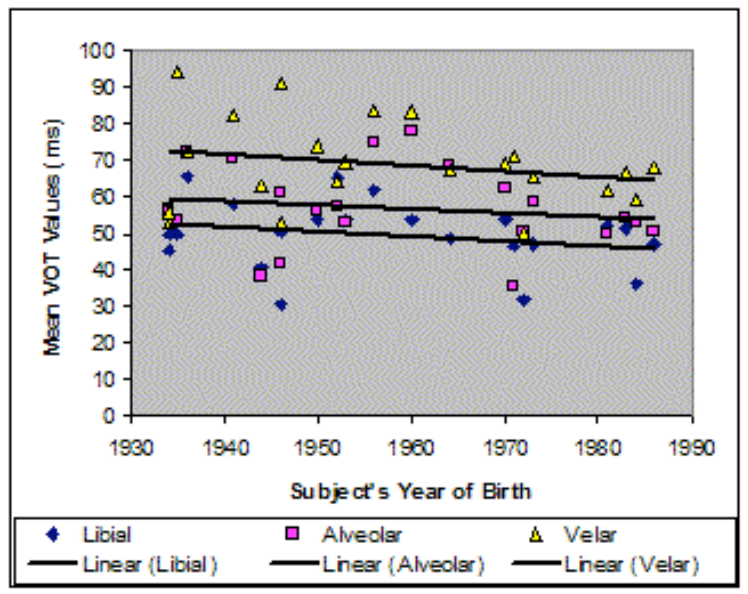

In terms of phonation type, similar shrinking pattern of VOT values was observed. As we see in Figure (3), the overall VOT range decreases for younger subjects. The lowest VOT value remains pretty much the same over time (around $15 \mathrm{~ms}$ ), but the highest VOT value has decreased from as high as $144.6 \mathrm{~ms}$ for older speakers to as low as $72.1 \mathrm{~ms}$ for younger speakers. Also, among the older participants, we see a little overlap between tense and lax stops, and greater overlap between lax and aspirated stops, but among younger participants, the overlap between lax and aspirated stops is substantial, with both being clearly distinct from tense stops. One young person, however, shows an overlap between tense and lax stops (see section 4).

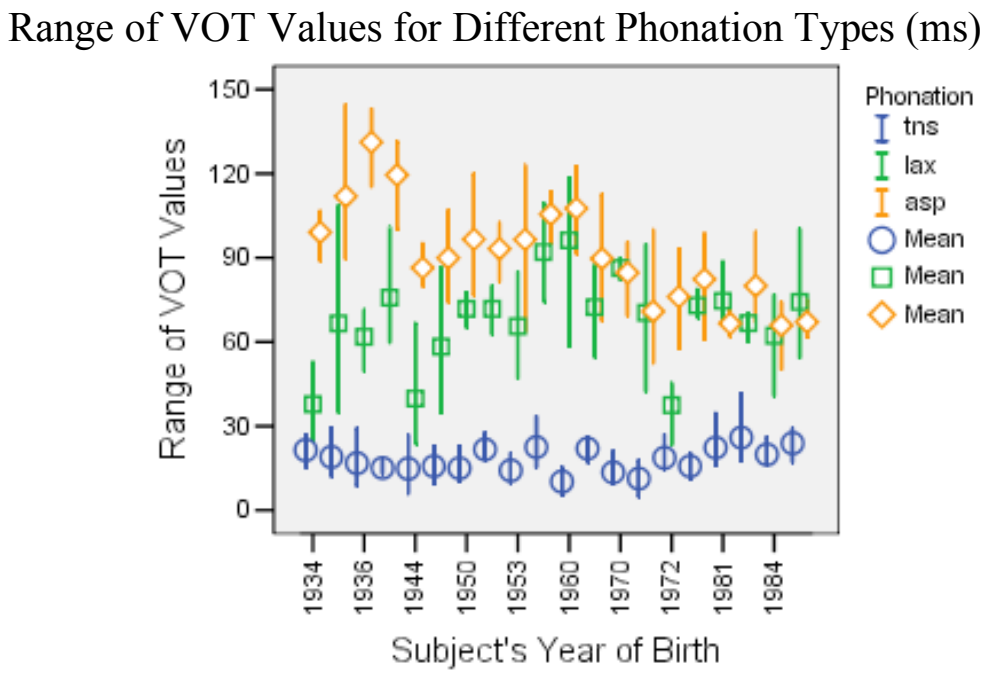


Wenhua Jin

To better capture the apparent shift over time, mean VOT values of the three phonation types for each subject were calculated and plotted at a function of each subject's year of birth (Figure (4)). For older speakers, the mean VOT value for the three phonation types are clearly distinct, with aspirated stops having higher VOT values than lax stops, which in turn show higher VOT values than tense stops. However, for subjects born in and after 1970, we see the emerging of a different pattern where mean VOT values of lax stops are almost as high as or even higher than those of aspirated stops, and there is a substantial overlap between mean VOT values of these two categories.

As indicated by the trend lines, mean VOT values of aspirated stops have decreased over time, while those of lax stops have increased, and those of tense stops remain basically stable. Thus, the change is basically one in the relative difference in mean VOT values between lax and aspirated stops, hereafter referred to as 'delta VOT': $\triangle \mathrm{VOT}=\mathrm{Mean}_{\mathrm{VOT}}$ asp - Mean $\mathrm{VOT}_{\text {lax }}$ (Silva 2006:293).

(4) Mean VOT Values for Different Phonation Type (ms)

(5) Distribution of $\triangle$ VOT (ms)
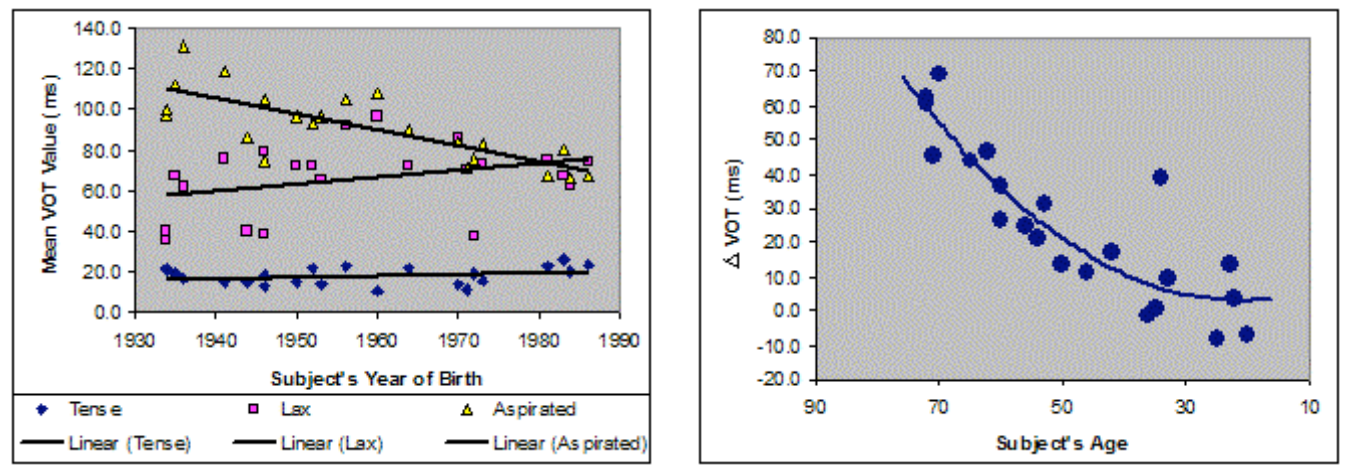

As illustrated in Figure (5), the general pattern observed here is that as subject's age gets younger, $\triangle \mathrm{VOT}$ becomes lower. More specifically, the difference in mean VOT values between aspirated and lax stops produced by older speakers could be as high as about $70 \mathrm{~ms}$, which continuously decreases to around $15 \mathrm{~ms}$ for middle aged people, reaching the lowest value in the young subjects' performance to negative values. The best fit curve for the data is Quadratic $(\mathrm{R} 2=0.797)$, indicating a non-linear relationship between $\Delta \mathrm{VOT}$ and subjects' age. Further hierarchical cluster analysis on the data divides the subjects into two major groups: subjects born before 1970 form an older group and subjects born in and after 1970 form a younger group, which matches well with what we observed above in Figure 3 about the point where the new pattern emerges. One subject born after 1970, however, behaves like older group, while two subjects born before 1970 patterns like younger group (see section 4).

To examine the difference between the two groups, mean VOT of three phonation types were calculated for each group. As illustrated in Figure (6), in older group, mean VOT values are significantly different for all three phonation 
types, while in younger group, although mean VOT values of tense stops are distinct from those of both lax and aspirated stops, no significant difference was observed between lax and aspirated stops ( $>00.05)$, suggesting a merger in VOT values between these two categories.

Mean VOT Values for Older and Younger Groups

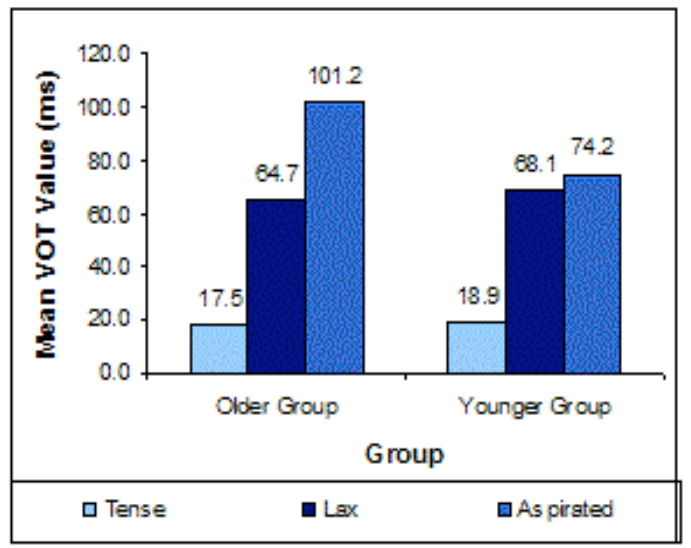

\subsection{Patterns of F0, Intensity Build-up, and Vowel Length}

In searching for potential acoustic cues for marking the underlying contrastive stops, F0 onset and offset, intensity build-up, and vowel length were also examined in this study.

For the younger group, both F0 onset and offset values of the vowel immediately following the target stop are highest after aspirated stops $\left(\mathrm{Mean}_{\text {onset }}\right.$ $\left.212 \mathrm{~Hz}, \mathrm{Mean}_{\text {offset }} 196 \mathrm{~Hz}\right)$, lowest after lax stops $\left(\right.$ Mean $_{\text {onset }} 159 \mathrm{~Hz}, \mathrm{Mean}_{\text {offset }}$ $156 \mathrm{~Hz}$ ) and medium after tense stops $\left(\mathrm{Mean}_{\text {onset }} 191 \mathrm{~Hz}, \mathrm{Mean}_{\text {offset }} 186 \mathrm{~Hz}\right)$. Similar findings were reported in previous studies on Seoul Korean speakers by Han and Weitzman (1970), Cho (1996), Han (1996), Silva (2004). Statistics analysis reveals a significant difference in F0 onset and offset values between after lax and after aspirated stops. The same pattern holds true for the older group. So throughout the data, F0 after aspirated stops constitute the highest register, while F0 after lax stops, the lowest register. As to F0 onset and offset after tense stops, for the younger group they are not significantly different from those after lax or after aspirated stops; for the older group, they are significantly different from those after lax stops, but not from those after aspirated stops.

In terms of intensity build-up, examination on the younger group shows that after aspirated stops, it takes shortest period of time (mean: 27ms) for the vowel to reach the fullest amplitude, while after lax stop, it takes much longer time (mean: $37 \mathrm{~ms}$ ), and the difference between the two time periods is statistically significant. After tense stops, it still takes significantly longer time $(39 \mathrm{~ms})$ than after aspirated stops, but is not significantly different from that of after lax stops. Again, the same pattern is observed in the older group. Thus, throughout the data, 


\section{Wenhua Jin}

intensity build-up is fastest after aspirated stops and significantly slower after lax and tense stops, with no apparent change over time.

In terms of vowel length, for the older group, the vowel length after all the three categories are significantly different from each other, with the vowel length after aspirated stop being the shortest $(61 \mathrm{~ms})$, that after tense stop the longest (126ms), and that after lax stop the medium (93ms). For the younger group, however, although the vowel after aspirated stop (62ms) is shorter than after lax vowel (73ms), they are not significantly different at $95 \%$ confidence interval, but they both are significantly shorter than the vowel after tense stop (105ms). Thus, vowel length does not provide itself as a consistent acoustic cue for the distinction of the underlying contrastive stops. ${ }^{2}$

\section{Discussion}

As early as in the 1960s, studies on Korean pointed out that VOT may well be the single most important measure for separating the three Korean stop categories (Lisker and Abramson 1964). Later studies in the 1990s (Silva 1992, Cho 1996, Han 1996) proved that VOT alone is sufficient to mark the distinction of the three phonation types of the stops. However, recent studies on Seoul Korean (Silva 2004, 2006) revealed a change in the role of VOT as the primary indicator, downgraded now to the secondary level with F0 being promoted to the primary level. Is this a unique phenomenon of Seoul Korean or is it that the Korean language as a whole is undergoing this change? Thus, studies on other Korean variety along the similar line may contribute to a better understanding of Korean language variation and change.

Acoustic and statistic analysis on the Chinese Korean data in this study reveals a diachronic shift in VOT values and a change in the role of VOT in separating the three stop categories in Chinese Korean. As illustrated in Figures (2) and (4) above, VOT values have shrunk over time both in terms of place of articulation and phonation types of the stops, though in the former case the distinction among the three categories are maintained while in the latter case there is a three to two-category VOT shift over time, i.e. while older people show clearer distinction in VOT values for the three phonation categories of stops, younger people born in and after 1970 manifest a merger in VOT values between aspirated and lax stops, suggesting that VOT alone may not be sufficient to mark the distinction of the stops. Further analysis shows that there may be several acoustic features that distinguish the different types of stops, and the relative importance of these features seems to vary from generation group to generation group. These features could be VOT, F0, intensity build-up, etc. For the older group under the current analysis, VOT plays the traditional primary role as documented in the literature; for the younger group, VOT still contributes to the distinction between tense stop and the other two categories, but there must be

\footnotetext{
${ }^{2}$ In this study, the consonant immediately following the vowel is not well controlled, which might contribute to the inconsistent conclusion about vowel length.
} 
another dimension that works with VOT in order to completely distinguish the three categories, and that is to further separate lax stops from aspirated ones. As analyzed above, for the younger group, F0 and intensity build-up consistently mark the difference between lax and aspirated stops, but not between tense and lax stops, which is a perfect complement of the role of VOT. Thus, for the younger group, VOT no longer plays a major role in stop distinction.

This change in VOT was better captured in Figure (5): oldest subjects show the greatest gap in VOT values between lax and aspirated stops, and as the age cohort gets younger, the observed gap becomes smaller, reaching the lowest value for the youngest subjects. Will this trend continue with even younger people than the youngest subjects in this study? The answer is no. As indicated by the best fit quadratic line, $\triangle \mathrm{VOT}$ for the youngest subjects in the study are stabilized around 0ms. Also, for the younger group, statistical analysis shows no correlation between $\triangle$ VOT and age, and every member of the group consistently show no significant VOT difference between lax and aspirated stops. Indeed, in another project of mine, six more subjects who are even younger show similar $\Delta$ VOT values as the youngest subjects under this analysis. Along a similar line, should more subjects be added who are older than the oldest subjects here, $\Delta$ VOT is not likely to go continuously higher and higher; rather $\Delta$ VOT will stabilize somewhere around the highest $\triangle \mathrm{VOT}$ value under this analysis. Thus, with a little bit of imagination, we can see the observed quadratic line is a component of an sshaped or z-shaped curve, which is often believed to be indicative of a language change in apparent time (Labov 1994, Guy 2003), with the assumption that different age groups available in the study reflect the language use of young adults of some time in the past.

Being the first systematic study on VOT of Chinese Korean, there is no previous data available for the current analysis to compare to, thus examination of the real time change is not possible at this stage. However, as been verified by many studies on other languages (e.g. Guy et al. 1986, Bailey et al. 1991), we believe this apparent time VOT shift phenomenon reflects the real time course of change in Chinese Korean. A good piece of side evidence, though, might be the similar VOT change pattern observed in Seoul Korean.

As discussed above, similar neutralization of VOT values was reported in the behavior of Seoul Korean speakers living in Dallas area of the United States (Silva 2004, 2006). The fact that the same pattern being observed in Chinese Korean, in a geographically remote area with completely different political and cultural settings may suggest that Korean language is undergoing this internal change. Meanwhile, we also noticed the existence of a different VOT pattern among young Chinese Korean speakers in Yánbiān area in China. Thus we have available three different locations where three different varieties of Korean show two different VOT patterns as illustrated in Figure (7): 
Wenhua Jin

VOT Patterns Observed in Young Speakers from Three Places ${ }^{3}$

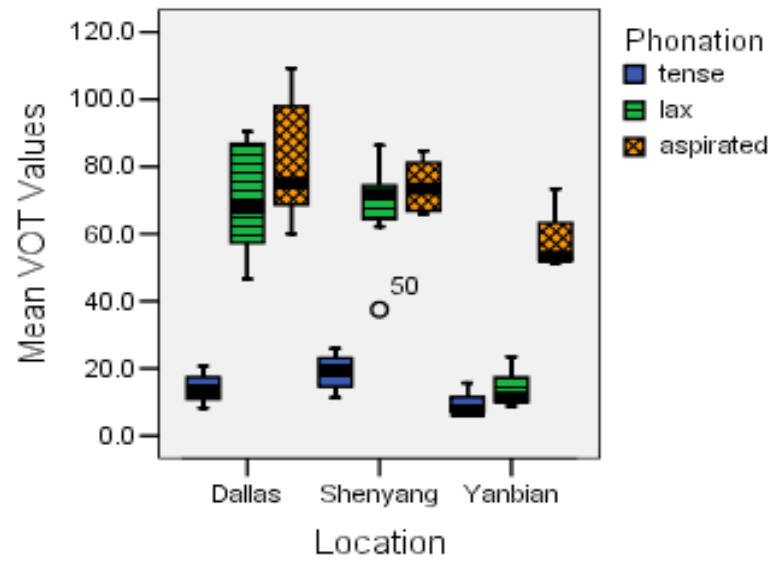

For Seoul Korean spoken in Dallas and Chinese Korean spoken in Shěnyáng, we see the latter is more advanced in the change as the mean VOT range of lax stops completely overlaps with that of aspirated stops, but they share the same pattern in that VOT overlap is between the lax and aspirated categories. For the Chinese Korean spoken in Yánbiān, however, the overlap is between tense and lax stops, with aspirated stops clearly distinct from the former two. We also notice that the mean VOT range of aspirated stops is much lower than the other two varieties. Overall, what they all have in common is that the aspirated stops of Korean are becoming less aspirated, and the three-way VOT distinction has shifted to a two-way distinction.

This general simplification shift may be explained as a result of language internal change to a universally less marked VOT system with the understanding that two-way VOT distinction is more common among world languages. Alternatively, this could be a result of language/dialect contact and influence from some social factors. Korean Chinese are often bilingual in Korean and Chinese. It is possible that Chinese, with its two-way VOT contrast, may influence in the long run the Korean production of Korean Chinese. Also, what has been noticed in this study is that three speakers do not pattern with their age groups: two subjects born before 1970 behaves like younger people, while one subject born after 1970 shows a pattern of older people. Analysis of these subjects' demographic data shows that the former two subjects are different from the rest of their group members in that they have great amount of access to Seoul Korean: one had worked in Korea for ten years, and the other visits Korea frequently. Should Seoul Korean speakers in Seoul speak the same way as the Seoul Korean speakers in Dallas area (still a pending issue due to lack of relevant study), then dialect contact may be a factor in the performance of the two subjects. As to the exceptional young subject, he is a Korean language teacher. His positive attitude

\footnotetext{
${ }^{3}$ The younger speakers included here are all born in and after 1970. Data source for the other two places are from Silva (2004), Zheng and Li (2005).
} 
towards Chinese Korean culture and language may have made him adhere to the traditional pattern, thus language attitude may also be a factor. As to the different VOT pattern between two varieties of Chinese Korean, a tentative explanation proposed here is that being the biggest Korean Prefecture in China, Yánbiān, unlike Shěnyáng, is like an isolated 'speech island' where the Korean language and culture are better preserved than anywhere else in China. Thus, the Korean variety spoken there may maintain the traditional VOT pattern as documented in Korean studies in 1960s and 70s, where the VOT overlap between tense and lax stops was observed. So, we conclude that VOT shift may not be a solely agerelated phenomenon; rather it may be a result of combined influence from several internal and external factors.

\section{Conclusion}

This study reveals a diachronic VOT shift in Chinese Korean and the change of the role of VOT as the primary marker of stop distinction. More specifically, it reports a merger in VOT values between lax and aspirated stops among younger speakers. Thus, while for older people VOT plays a primary role in separating the stop types, for younger people it may need to work together with other dimensions in the grammar, i.e. F0, intensity build-up, to fulfill the task. Further comparison of different VOT change patterns indicates that across dialects Korean aspirated stops are becoming less aspirated, and VOT is shifting towards a simpler two-way system. These changes may be explained as a result of combined influence from several internal and external factors.

Further studies will need to systematically incorporate more sociolinguistic factors beyond age, and continue with analysis on other Korean varieties spoken in China and also in other countries such as Korea itself, Japan, Russia, Spain, etc. to determine the direction of change and better understand the reasons and the course of Korean language change.

\section{References}

Bailey, Guy, Tom Wikle, Jan Tillery, and Lori Sand. 1992. The Apparent Time Construct. Language Variation and Change. Cambridge University Press, 3:241-264.

Cho, Taehong. 1996. Vowel Correlates to Consonant Phonation: An AcousticPerceptual Study of Korean Obstruents. MA thesis, University of Texas at Arlington.

Guy, Gregory R. 1981. Linguistic Variation in Brazilian Portuguese: Aspects of the Phonology, Syntax, and Language History. Ph.D. Dissertation, University of Pennsylvania.

Guy, Gregory R. 2003. Variationist Approaches to Phonological Change. The Handbook of Historical Linguistics. Brian D. Joseph and Richard D. Janda, eds., Blackwell Publishing, 367-400. 
Wenhua Jin

Han, Jeong-Im. 1996. The Phonetics and Phonology of 'Tense' and 'Plain' Consonants in Korean. Ph.D. dissertation, Cornell University. Distributed 1996, Ithaca: CLC Publications.

Han, Mieko S. and Raymond S. Weitzman. 1965. Studies in the Phonology of Asian Languages III: Acoustic Characteristics of Korean Stop Consonants. Los Angeles: Acoustic Phonetics Research Laboratory, University of Southern California.

Han, Mieko S. and Raymond S. Weitzman. 1970. Acoustic Features of Korean /P, $\mathrm{T}, \mathrm{K} /, / \mathrm{p}, \mathrm{t}, \mathrm{k} /$ and $/ \mathrm{ph}$, th, kh/. Phonetica 22:112-128.

Kim, Chin-W. 1965. On the Autonomy of the Tensity Feature in Stop Classification (with Special Reference to Korean Stops). Word 21:339-359.

Labov, William. 1994. Principles of Language Change: Internal Factors. Oxford \& Cambridge, Mass.: Blackwell.

Ladefoged, Peter. 2003. Phonetic Data Analysis: An Introduction to Fieldwork and Instrumental Techniques. Malden, Mass. \& Oxford: Blackwell.

Lisker, Leigh and Arthur S. Abramson. 1964. A Cross-Language Study of Voicing in Initial Stops: Acoustical Measurements. Word 20:384-422.

Silva, D.J. 1992. The Phonetics and Phonology of Stop Lenition in Korean. PhD Dissertation, Cornell University. Distributed 1992, Ithaca: CLC Publications.

Silva, D. J, Younjeoung Choi and Ji Eun Kim. 2004. Evidence for a Diachronic Shift in the VOT Values of Korean Stop Consonants. Susumu Kuno et al. eds., Harvard Studies in Korean Linguistics $X$. Cambridge, MA: Harvard University, Department of Linguistics, 173-185.

Silva, D.J. 2006. Acoustic Evidence for the Emergence of Tonal Contrast in Contemporary Korean. Phonology 23:287-308. Cambridge University Press.

Silva, D.J. and Wenhua Jin. 2006. Chinese Korean Segmental Phonetics: A Field Report. Harvard Studies In Korean Linguistics XI. Susumu Kuno et al. eds., Cambridge, MA: Harvard University, Department of Linguistics, Hanshin Publishing Company, 264-277.

郑鲜日, 李英浩. 2005. 英, 朝塞音的浊音起始时间（VOT）对比及朝鲜族 学生习得英语塞音特征研究. 延边大学学报 第38卷, 第4期.

赵习, 宣德五. 1986. 朝鲜语六镇话的方言特点, 民族语文 第5期，第1-13 页。

최윤갑, 전학석. 1993. 중국, 조선, 한국에서의 조선어차이에 대한. 연변인민출판사.

Wenhua Jin

University of Texas at Arlington

Department of Linguistics and TESOL

Hammond Hall 403

Arlington, TX, 76019

jinwenh@hotmail.com 